\title{
Vibroacoustic influence use in training young boxers
}

\author{
Albert Khasanov ${ }^{1}$, Elvira Shayakhmetova ${ }^{1 *}$, Rita Jamileva ${ }^{1}$, Ludmila Matveeva ${ }^{1}$, Stanislav Matveev ${ }^{2}$ \\ ${ }^{1}$ Bashkir State Pedagogical University named after M. Akmulla, 450008, Ufa, Russia \\ ${ }^{2}$ Bashkir State University, 450076, Ufa, Russia
}

\begin{abstract}
The article presents mobility and balance of nervous processes analysis in young boxers during vibroacoustic influence use. The research was held on the basis of Republican boarding school N5 of sports profile in Ufa. 82 boxers took part in the research (14-15 years old). The respondents were divided into two groups: control group $(n=48)$ and the experimental group $(n=34)$. General mobility, balance and power of nervous processes estimation was held at psychophysiological testing gadget "Sports psychophysiologist". As the means of rehabilitation we used tinting with the help of medical apparatus "Vitophone". Young boxers' calf muscles tinting was held in the following regimen: twice (10-15 minutes each) at the end of the first daily training lesson of special-preparatory period. During microvibrations use at the end of the first training lesson we reveal nervous system excitability in young boxers, the power of nervous processes also increases in terms of external disturbances, the accuracy of reactions increases, there is balance of irritation and inhibition. As a result of the research we revealed the possibilities of vibroacoustic influence use in the process of young boxers training.
\end{abstract}

\section{Introduction}

Scientific resources and athletes' sports training process analysis showed high interest of scientists in searching new approaches. They increase organism resistance to tiredness. They provide working capacity improvement and intensifying the processes of athletes' rehabilitation, improving psycho-emotional state [ 1-6].

In the present research work tinting with the help of medical apparatus "Vitophone" was used as the means of rehabilitation. This apparatus is used for vibroacoustic physiotherapy (the author of the method and the device is a scientist physicist -inventor V. A. Fedorov, registration certificate № FSR 2010/07778, Russia).

The definite audio frequency microvibrations influence regulating function of nervous system. It conditions the work of different systems and organs. An adequate functioning of CNS is based on blood flow increase in capillaries, vascular blood flow resistance, its circulating volume increase owing to stagnant part in depot. It is involved into bloodstream under the influence of vibrations [7].

The aim of the research is to define mobility and balance of nervous processes in young boxers during vibroacoustic influence use.

\section{Materials and Research Methods}

The research was held on the basis of Republican boarding school N5 of sports profile in Ufa, Russia. 82 boxers took part in the research (14-15 years old). The respondents were divided into two groups: the control group ( $\mathrm{n}=48)$ and the experimental group $(\mathrm{n}=34)$.

Training lessons were held every day (apart from Sunday), twice a day three academic hours. Young men of the control and the experimental groups went through calf muscles tinting in the regimen twice 10-15 minutes each at the end of the first daily training lesson of special-preparatory period. 2 regimen choice (from $200 \mathrm{~Hz}$ till $18 \mathrm{kHz}$ ) was conditioned by athletes' mass and the place of tinting. Vibraphone was set by membranes to the body in the sitting position. During the days of rest tinting was not held.

The following research methods were used in the work: literature sources analysis, stating and forming experiments, mathematical-statistical analysis. General mobility of nervous processes, balance (standard deviation) and power (accuracy value) of nervous processes were studied with the help of difficult visual-motor reaction indices, choice reaction (DVMR, CR). Balance of nervous processes was estimated with the help of "Reaction to a moving object" (RMO) methodology. These indices study was held at the device of psycho-physiological testing "Sports psychophysiologist".

Mathematical-statistical handling was held with the help of Microsoft Exsel 2007 and program package Attestat

\footnotetext{
*Corresponding author: shaga.elv@yandex.ru
} 
using generally adopted methods of variation statistics. During mathematical handling we calculated the following: arithmetical mean of the testing results $(\mathrm{M})$; mean square deviation (m); Student t-test.

\section{Results and Discussion}

Mobility of nervous processes estimation, held with the help of "choice reaction" methodology showed that the initial average group parameters of CNS activity in young boxers have statistically valid differences in accordance with normative CR indices (in case $\mathrm{p}=0,001)$ (table 1 ).

Boxing is characterized by direct physical contact between athletes. High index of defense speed or attack techniques use defines favorable position of each opponent. In this case young athletes' state in both groups means slow "warming-up".

Table 1. The indices of nervous processes mobility in young boxers in special-preparatory period in terms of vibroacoustic influence $(\mathrm{M} \pm \mathrm{m}), \mathrm{n}=82$

\begin{tabular}{|c|c|c|c|c|c|}
\hline \multirow{2}{*}{ Indices } & \multirow{2}{*}{ Groups } & \multirow{2}{*}{ Initial results } & \multicolumn{3}{|c|}{ Micro cycles } \\
\hline & & & 1 & 2 & 3 \\
\hline \multirow{2}{*}{$\begin{array}{l}\text { DVMR (CR), } \\
\text { ms } \\
\text { Norm: } 266,2 \pm 8,5 \\
\text { ms }\end{array}$} & $\mathrm{CG}$ & $289,4 \pm 9,2$ & - & - & $270,2 \pm 4,2 *$ \\
\hline & EG & $287,5 \pm 9,3$ & $282,5 \pm 7,6^{*}$ & $270,0 \pm 6,3^{*}$ & $267,2 \pm 2,8 *\lrcorner$ \\
\hline
\end{tabular}

Notes: DVMR - difficult visual-motor reaction, CR - choice reaction, * - validity of differences between the initial and further indices, ${ }_{-}=-$- validity of differences between indices of the EG and CG.

It should be noted that by the end of the $1^{\text {st }}$, the $2^{\text {nd }}$ and the $3^{\text {rd }}$ weekly micro cycles in boxers from the experimental group there were valid differences of the studied parameter average values. The dynamics of reaction time proves high level of nervous processes power in terms of external interference. High percentage of reaction accuracy validity proves complete attention concentration. In boxing the athlete should master quick "recognition" of opponent's defrauding manoeuvres and should be able to react quickly.

Mobility of nervous processes in CNS and a high level of nervous processes power in terms of external interference is the base for quick reaction to tactical techniques during sparing. Validity level of reactions accuracy proves that athletes can concentrate when it is necessary during the combat. We consider that these changes happened as a result of microvibrations influence in boxers from the EG.

Boxers from the CG also had validly significant differences of DVMR index at the end of three micro cycles of special-preparatory period. However, mobility index of nervous processes didn't achieve normative values.

The accuracy of reaction, balance of irritation and inhibition processes estimation was held with the help of "Reaction to moving object" (RMO) methodology. Balance degree analysis of irritation and inhibition processes in young boxers during special-preparatory period in terms of vibroacoustic influence is presented in table 2.

Table 2. The indices of nervous processes balance in young boxers during special-preparatory period of vibroacoustic influence $(\mathrm{M} \pm \mathrm{m}), \mathrm{n}=82$

\begin{tabular}{|c|c|c|c|c|c|}
\hline \multirow{2}{*}{ Indices } & \multirow{2}{*}{ Group } & \multirow{2}{*}{ Initial results } & \multicolumn{3}{|c|}{ Micro cycles } \\
\hline & & & 1 & 2 & 3 \\
\hline \multirow{2}{*}{$\begin{array}{l}\text { RMO } \\
\text { points) }\end{array}$} & $\mathrm{CG}$ & $9,8 \pm 0,4$ & - & - & $11,9 \pm 0,3^{*}$ \\
\hline & EG & $9,4 \pm 0,6$ & $10,6 \pm 0,5$ & $11,5 \pm 0,4^{*}$ & $14,2 \pm 0,4 *\lrcorner$ \\
\hline \multirow{2}{*}{$\begin{array}{l}\mathrm{RMO} \text { (retarded } \\
\text { reactions, ms) }\end{array}$} & CG & $2161,2 \pm 4,6$ & - & & $1713,0 \pm 3,6$ \\
\hline & EG & $2162,0 \pm 4,6$ & $1823,0 \pm 4,4^{*}$ & $1695,0 \pm 4,2 *$ & $1532,2 \pm 3,8^{*\lrcorner}$ \\
\hline \multirow{2}{*}{$\begin{array}{l}\text { RMO (anticipatory } \\
\text { reactions, } \mathrm{ms} \text { ) }\end{array}$} & $\mathrm{CG}$ & $-577, \pm 6,4$ & - & - & $-713,0 \pm 0,8 *$ \\
\hline & EG & $-570, \pm 4,9$ & $-428,0 \pm 3,2 *$ & $-361,0 \pm 1,9 *$ & $-267,0 \pm 1,6^{*}$ \\
\hline
\end{tabular}

Notes: RMO - reaction to moving object, * - validity of differences between the initial and further indices, $I^{\lrcorner}$I-validity of differences between indices of the EG and the CG.

The results in table 2 prove that in the initial state accuracy, retarded and anticipatory reactions in young athletes from two groups didn't have validly significant differences. Starting from the $2^{\text {nd }}$ weekly micro cycle the athletes from the experimental group (in terms of vibroacoustic influence) had the increase of the accurate reactions amount. 
In the average all examined athletes had balanced nervous system. However, for the athletes of the experimental group it was typical to have the tendency of inhibition processes prevalence, which can prove self -control, selfrestraint and cold-bloodedness.

Lack of balance, owing to inhibition prevalence over irritation, conditions caution, self-restraint and calm behavior prevent risk and excitement.

\section{Conclusions}

Microvibrations of the definite audio frequency influence the regulating function of nervous system during calf muscles tinting in regimen twice 10-05 minutes each at the end of the first daily training lesson during specialpreparatory period.

During microvibrations use at the end of the first training lesson we reveal nervous system excitability in young boxers. The power of nervous processes also increases in terms of external disturbances, the accuracy of reactions increases. There is balance of irritation and inhibition.

\section{References}

1. A.F. Gaynullina, A.L. Lintvarev, E.Sh. Shayakhmetova, Pedagogico-psychological and medico-biological problems of physical culture and sport, 3, 237-244 (2017

2. A.F. Khaervarina, L.M. Matveeva, R.M. Muftakhina, E.Sh. Shayakhmetova, Theory and practice of physical culture, 8, 73-75 (2019)

3. E.R. Khakimov, A.F. Khaervarina, A. T. Khasanov, Psychophysiology bulletin, 3, 118-123 (2018)

4. M.K. Beauchamp, R.H. Harvey, P. Beauchamp, J Clin Sport Psychol, 6, 67-84 (2012)

5. H.A. Hashim, Asian J Sports Med, 2, 99-105 (2011)

6. L. Shaw, L. Zaichkowsky, V. Wilson, J Clin Sport Psychol, 6, 47-66 (2012).

7. L.I. Zhevnerchuk, L.N. Leonova, O.V. Arkhipova, Vibroacoustic effects influence on functional readiness of athletes: report of Scientific Research Work (City medical-physical culture clinic, Saint-Petersburg, 2003) 